\title{
Fatores que dificultam o aleitamento materno na Unidade de Terapia Intensiva
}

\section{Neonatal}

\author{
Factors that hinder beastfeeding in the Neonatal Intensive Care Unit \\ Factores que dificultan la lactancia materna em la Unidad de Cuidados Intensivos Neonatales
}

Recebido: 15/06/2021 | Revisado: 20/06/2021 | Aceito: 02/07/2021 | Publicado: 14/07/2021

Maiellen Pinheiro de Oliveira

ORCID: https://orcid.org/0000-0002-7235-6941

Centro Universitário Jorge Amado, Brasil

E-mail: maiellen18@gmail.com

Andreza Camardelli Rodrigues

ORCID: https://orcid.org/0000-0002-0582-2957

Centro Universitário Jorge Amado, Brasil

E-mail: dedacamardelli@gmail.com

Briane Schettini de Souza Corrêa

ORCID: https://orcid.org/0000-0002-6289-2695

Centro Universitário Jorge Amado, Brasil

E-mail: brianeschettinisc@gmail.com

Camila Trindade dos Santos Dias

ORCID: https://orcid.org/0000-0002-2937-4597

Centro Universitário Jorge Amado, Brasil

E-mail: camila-tsd@hotmail.com

Vitoria Vilas Boas da Silva Bomfim

ORCID: https://orcid.org/0000-0003-4897-0279

Centro Universitário Jorge Amado, Brasil

E-mail: pesquisaclinica9@gmail.com

Bruna Barreto Mascarenhas

ORCID: https://orcid.org/0000-0002-9614-744X

Universidade do Estado da Bahia, Brasil

E-mail: brunnamasc@outlook.com

Milena Oliveira Brito da Silva

ORCID: https://orcid.org/0000-0001-5935-6974

Universidade do Estado da Bahia, Brasil E-mail: milenaobs@outlook.com

Larissa Araújo Torres

ORCID: https://orcid.org/0000-0003-3235-5802 Universidade de Brasília, Brasil

E-mail: larissaa.torres@hotmail.com

Gleice Kelly Calixto Solidade

ORCID: https://orcid.org/0000-0001-8977-2733

Centro Universitário Anhanguera, Brasil

E-mail: gleicekellymarques@gmail.com

Gabrielly Albuquerque Pinho

ORCID: https://orcid.org/0000-0002-2729-2643 Universidade de Brasília, Brasil

E-mail: gabriellya056@gmail.com

Vyttória de Oliveira Albuquerque

ORCID: https://orcid.org/0000-0001-7242-4118

Centro Universitário Tiradentes, Brasil

E-mail: vyttoriaalbuquerque@hotmail.com

Stefany de Oliveira Chaves

ORCID: https://orcid.org/0000-0001-5902-2472

Centro Universitário Tiradentes, Brasil

E-mail: stefanyochaves@gmail.com

Paulo Ricardo Lima Bastos

ORCID: https://orcid.org/0000-0001-6878-1075

Centro Universitário Maurício de Nassau, Brasil

E-mail: pauloricardobastos2014@gmail.com

\section{Resumo}

Introdução: O leite materno constitui a principal e a melhor fonte de alimentação para uma criança que esteja em fase de desenvolvimento. Porém, quando um bebê vai para uma unidade de terapia intensiva neonatal, é muito comum que 
a mãe tenha dificuldade de oferecer o leite materno devido a vários fatores. Objetivo: Identificar os fatores que dificultam o aleitamento materno na UTI Neonatal. Metodologia: Trata-se de uma revisão integrativa da literatura realizada entre Setembro à Outubro de 2020 nas bases de dados: Banco de Dados de Enfermagem (BDENF), Literatura Latino-americana e do Caribe em Ciências da Saúde (LILACS) e Sistema Online de Busca e Análise de Literatura Médica (MEDLINE), com artigos publicados entre os anos 2015 a 2019. Resultados: A busca evidenciou oito artigos para compor o estudo, no período de 2015 a 2019, disponíveis na íntegra na língua inglesa e portuguesa. As principais categorias encontradas foram: Sentimentos das mães quanto ao recém-nascido internado em UTIN, as experiências maternas relacionadas à amamentação em uma UTIN e a importância do apoio da equipe profissional e da família relacionada à amamentação. Considerações Finais: Foi possível analisar que em um cenário de uma UTIN existe várias fragilidades em relação ao aleitamento materno, dentre elas são: A falta de apoio dos profissionais, sentimentos negativos das mães e suas experiências relacionadas ao ato de amamentar. É muito importante que o profissional de saúde seja cauteloso, incentivador e um facilitador para que essa mãe siga firme nos cuidados do seu bebê.

Palavras-chave: Aleitamento materno; Récem-nascido; Unidade de Terapia Intensiva Neonatal.

\begin{abstract}
Introduction: Breast milk is the main and the best source of food for a child who is in the development stage. However, when a baby goes to a neonatal intensive care unit, it is very common for the mother to have difficulty offering breast milk due to several factors. Objective: To identify the factors that hinder breastfeeding in the Neonatal ICU. Methodology: This is an integrative literature review carried out between September and October 2020 in the databases: Nursing Database (BDENF), Latin American and Caribbean Literature on Health Sciences (LILACS) and Online System of Search and Analysis of Medical Literature (MEDLINE), with articles published between the years 2015 to 2019. Results: The search revealed eight articles to compose the study, from 2015 to 2019, available in full in English and Portuguese. The main categories found were: Mothers' feelings about the newborn admitted to the NICU, maternal experiences related to breastfeeding in a NICU and the importance of support from the professional team and the family related to breastfeeding. Final Considerations: It was possible to analyze that in a setting of a NICU there are several weaknesses in relation to breastfeeding, among them are: The lack of support from professionals, negative feelings of mothers and their experiences related to the act of breastfeeding. It is very important that the health professional be cautious, supportive and a facilitator so that this mother remains firm in the care of her baby.
\end{abstract}

Keywords: Breastfeeding; Newborn; Neonatal Intensive Care Unit.

\title{
Resumen
}

Introducción: La leche materna es la principal y mejor fuente de alimento para un niño que se encuentra en etapa de desarrollo. Sin embargo, cuando un bebé va a una unidad de cuidados intensivos neonatales, es muy común que la madre tenga dificultades para ofrecer leche materna debido a varios factores. Objetivo: Identificar los factores que dificultan la lactancia materna en la UCI Neonatal. Metodología: Se trata de una revisión bibliográfica integradora realizada entre septiembre y octubre de 2020 en las bases de datos: Base de Datos de Enfermería (BDENF), Literatura Latinoamericana y del Caribe en Ciencias de la Salud (LILACS) y Sistema Online de Búsqueda y Análisis de Literatura Médica (MEDLINE), con artículos publicados entre los años 2015 a 2019. Resultados: La búsqueda reveló ocho artículos para componer el estudio, de 2015 a 2019, disponibles en su totalidad en inglés y portugués. Las principales categorías encontradas fueron: Sentimiento de las madres sobre el recién nacido ingresado en la UCIN, experiencias maternas relacionadas con la lactancia materna en una UCIN y la importancia del apoyo del equipo profesional y familiar relacionado con la lactancia. Consideraciones Finales: Se pudo analizar que en el ámbito de una UCIN existen varias debilidades en relación a la lactancia materna, entre ellas se encuentran: La falta de apoyo de los profesionales, los sentimientos negativos de las madres y sus vivencias relacionadas con el acto de amamantar. Es muy importante que el profesional de la salud sea cauteloso, comprensivo y facilitador para que esta madre se mantenga firme en el cuidado de su bebé.

Palabras clave: Lactancia materna; Recién nacido; Unidad de Cuidados Intensivos Neonatales.

\section{Introdução}

O leite materno é a principal e melhor fonte de alimento para uma criança durante a fase de desenvolvimento, pois a sua produção é adaptada de acordo com todas as necessidades nutricionais desta criança (Brasil, 2019). Contudo, quando um recém-nascido (RN) vai para uma Unidade de Terapia Intensiva Neonatal (UTIN) surgem diversos obstáculos que dificultam a oferta desse leite, sendo um deles a quebra do binômio mãe e filho (Teston et al., 2018).

De acordo com os autores Morais et al (2020), outro fator determinante é o esgotamento físico e emocional da nutriz, oriundo de um internamento prolongado, resultando em uma baixa produção de leite. Por outro lado, esse RN pode apresentar uma sucção débil que impossibilitará o esvaziamento completo da mama, podendo em alguns casos dificultar também o início 
da amamentação (Pereira et al., 2015). Além disso, conforme referido por Lopes et al (2015), dispneia durante a mamada, sonolência e diminuição do reflexo de busca também se tornam obstáculos para uma amamentação eficaz.

Conforme afirma Valadares (2016), entre os anos de 1990 a 2014 ocorreu uma queda significativa na mortalidade infantil em crianças menores de cinco anos no Brasil, passando de 66 casos para 12,9 para cada mil nascidos vivos, tudo isso graças aos esforços no incentivo ao aleitamento materno. Porém, estudos apontam a dificuldade da manutenção desse aleitamento após alta hospitalar, principalmente em recém-nascidos pré-termo (RNPT), tendo como fator principal para interrupção a ideia de que o leite não é suficiente para nutrir a criança (Lima et al., 2019). RNs com idade gestacional <32 semanas, possuem maior risco de descontinuação da amamentação quando comparados a prematuros com idade gestacional superior há 32 semanas (Freitas et al., 2015).

Marson (2015) relata que muitas das vezes, por conta do quadro clínico do RN, o início da amamentação é adiado, sendo necessária a administração de Nutrição Parenteral (NPT), podendo também, em alguns casos, fazer uso de sonda nasogástrica, sendo utilizado o leite ordenhado pela própria nutriz. Apesar de ser possível a oferta desse leite através da ordenha, a falta de apoio e aconselhamento aumenta a chance de um desmame precoce, afinal, o ambiente hostil influencia diretamente na produção do leite. O desmame, segundo o Ministério da Saúde (2015), pode provocar o surgimento do ingurgitamento mamário, estase do leite, mastite, tristeza e depressão devido à perda do leite materno ou mudanças hormonais.

$\mathrm{O}$ enfermeiro precisa ser um facilitador às mulheres-nutriz, promovendo por meio do diálogo, o apoio e aconselhamento, buscando garantir o sucesso da amamentação mesmo após a alta hospitalar. Desta forma, com as orientações iniciadas ainda durante o internamento, podem contribuir para abolir incertezas, superar obstáculos, prevenir futuros problemas mamários e garantir o sucesso da posição do bebê e da pega correta (de Souza Baptista et al., 2015).

Considerando os fatores que dificultam o aleitamento materno em uma UTIN, é imprescindível compreender quais são estes fatores e qual a melhor maneira de reduzir os impactos do desmame precoce nos neonatos. Na enfermagem, a pesquisa sobre o tema supracitado, faz com que o atendimento ao lactente e sua genitora se torne mais humanizado e permite que sejam criados e implementados novos programas, possibilitando assim a redução do índice de desmame precoce em unidades hospitalares, promovendo o contato mãe-bebê, evitando o aparecimento de doenças crônicas e proporcionando a maturidade do sistema imunológico da criança.

Diante do proposto a cima, tem-se a seguinte questão de pesquisa: Quais são os principais fatores que dificultam o aleitamento materno em uma UTI neonatal? O objetivo deste estudo é identificar fatores que possam dificultar o aleitamento materno em uma unidade de terapia intensiva neonatal.

\section{Metodologia}

Trata-se de uma revisão integrativa da literatura, definida por Souza et al. (2010) como um instrumento que utiliza a síntese de conhecimentos e aplicação de resultados de estudos e/ou pesquisas de um determinado assunto.

A coleta de dados ocorreu em Setembro à Outubro de 2020 e norteou-se pela seguinte pergunta de pesquisa: Quais os fatores que dificultam o aleitamento materno em uma UTI neonatal? O local de busca foi a Biblioteca Virtual em Saúde (BVS) nas bases de dados: Banco de Dados de Enfermagem (BDENF), Literatura Latino- americana e do Caribe em Ciências da Saúde (LILAC'S) e Sistema Online de Busca e Análise de Literatura Médica (MEDLINE) e houve a utilização dos seguintes descritores, consultados na plataforma dos Descritores em Ciências da Saúde (DeCS): "aleitamento materno", "recémnascido" e "unidade de terapia intensiva neonatal". A combinação dos descritores ocorreu através do operador booleano "AND". 
Os critérios de inclusão se definiram como artigos disponíveis na íntegra, publicados em português e inglês nos últimos 05 anos (2015 - 2019). Excluídos aqueles duplicados nas bases e os quais não responderam à pergunta de pesquisa acima exposta, teses, protocolos, dissertação, revisão e artigos pagos.

Não houve necessidade de submeter o estudo ao Comitê de Ética, conforme a Resolução 466/2012 do Conselho Nacional de Saúde. Contudo, será devidamente respeitada, conforme Lei $\mathrm{N}^{\circ}$ 9.610, de fevereiro de 1998, toda a autoria do que for citado (Brasil, 2013).

\section{Resultados}

Após a inclusão dos descritores, foram identificados 189 artigos na língua portuguesa e inglesa, publicados entre os anos de 2015 a 2019, disponíveis na íntegra em português e inglês. Depois da leitura dos títulos e análise dos resumos, foram excluídos 15 artigos por publicação duplicada, sendo: sete na base de dados BDENF e oito na LILACS, 160 devido à incompatibilidade com o objetivo da pesquisa, sendo: 150 na base de dados MEDLINE, 9 na LILACS e 1 na BDENF. Além disso, foram excluídos seis artigos na MEDLINE que estavam indisponíveis na íntegra. Seguindo os critérios de inclusão, oito artigos foram selecionados para compor o estudo.

Quanto ao tipo de estudo, um é estudo de coorte retrospectivo, quatro são estudos transversais e três são estudos qualitativos, dos quais um também é descritivo. Foi possível constatar que seis artigos foram publicados em periódicos estrangeiros, dos quais são: BMC Pediatrics, Advances in Neonatal Care Journals, Breastfeed Med, International Breastfeedin Journals, Journal of Human Lactation e Hospital Pediatrics, sendo um estudo para cada periódico e dois artigos são de periódicos nacionais, sendo um da Revista de Enfermagem Escola Anna Nery e um da Revista Distúbios da Comunicação.

Os países de origem dos artigos selecionados estavam assim distribuídos: Um da Itália, um do Canadá, dois dos Estados Unidos (EUA), dois do Brasil, um de Pequim e um de Portugal.

No que se refere aos anos de publicação dos artigos, com recorte temporal entre 2015 e 2019, observa-se que as maiores concentrações de estudos foram nos anos de 2015, 2016 e 2018, com dois artigos cada ano, seguido dos anos de 2017 e 2019 com um artigo por ano.

Diante das análises dos estudos, foi possível comparar e reunir os artigos que apresentavam similaridade de conteúdo, caracterizando em três categorias para a temática: Sentimentos das mães quanto ao recém-nascido internado em UTIN, Experiências maternas relacionadas à amamentação em uma UTIN; A importância do apoio da equipe profissional e da família relacionada à amamentação.

Assim, o Quadro 1 representa a síntese dos artigos selecionados quanto aos autores/ano, país, periódico, tipo de estudo e os resultados encontrados. 
Quadro 1 - Síntese dos artigos selecionados quanto a autores/ano, país, periódico, tipo de estudo e os resultados encontrados. Brasil. 2021.

\begin{tabular}{|c|c|c|c|c|}
\hline Estudo & Autor/Ano & Periódico & $\begin{array}{l}\text { Tipo de } \\
\text { Estudo }\end{array}$ & Resultado do Estudo \\
\hline 01 & $\begin{array}{l}\text { Yang et al. } \\
\text { (2019) }\end{array}$ & $\begin{array}{c}\text { International } \\
\text { Breastfeeding } \\
\text { Journals }\end{array}$ & $\begin{array}{l}\text { Pesquisa } \\
\text { Qualitativa }\end{array}$ & $\begin{array}{l}\text { Ansiedade materna; Falta de apoio profissional; Falta } \\
\text { de interesse em amamentar por achar que o leite } \\
\text { materno não alimenta o recém-nascido; } \\
\text { Exaustão materna. }\end{array}$ \\
\hline 02 & $\begin{array}{l}\text { Bujold et al. } \\
\quad \text { (2018) }\end{array}$ & $\begin{array}{l}\text { Advances in } \\
\text { Neonatal Care } \\
\text { Journals }\end{array}$ & $\begin{array}{l}\text { Estudo } \\
\text { transversal; } \\
\text { Pesquisa } \\
\text { qualitativa e } \\
\text { descritiva }\end{array}$ & $\begin{array}{c}\text { Separação física entre mãe e bebê causava tristeza e } \\
\text { solidão; Desconforto materno e pressão em relação à } \\
\text { ordenha do leite; Desmotivação e frustração por não } \\
\text { conseguir amamentar/ordenhar e ter o contato com o } \\
\text { recém-nascido. }\end{array}$ \\
\hline 03 & $\begin{array}{l}\text { Gianni et al. } \\
\quad(2018)\end{array}$ & BMC Pedatrics & $\begin{array}{l}\text { Estudo } \\
\text { transversal; } \\
\text { Pesquisa } \\
\text { qualitativa }\end{array}$ & $\begin{array}{l}\text { Dificuldade em bombear o leite materno; ter um } \\
\text { recém- nascido de baixo peso e/ou com } \\
\text { comorbidades; ansiedade e o estresse materno; Dor } \\
\text { durante a extração do leite e falta de apoio pelos } \\
\text { profissionais de saúde. }\end{array}$ \\
\hline 04 & $\begin{array}{l}\text { Rossman et al. } \\
\text { (2017) }\end{array}$ & Breastfeed Med & $\begin{array}{c}\text { Estudo } \\
\text { Qualitativo }\end{array}$ & $\begin{array}{l}\text { Mães adolescentes relatam medo de julgamentos, } \\
\text { problemas com imagem corporal, pensamentos } \\
\text { desorganizados sobre o bombeamento do leite com a } \\
\text { volta da rotina escolar. }\end{array}$ \\
\hline 05 & $\begin{array}{l}\text { Alves } e t \text { al. } \\
\text { (2016) }\end{array}$ & $\begin{array}{c}\text { Journal of } \\
\text { Human Lactation }\end{array}$ & $\begin{array}{c}\text { Estudo } \\
\text { Transversal } \\
\text { Quantitativo. }\end{array}$ & $\begin{array}{l}\text { Preocupações com a oferta inadequada do leite; } \\
\text { Dificuldades para tirar o leite; } \\
\text { Separação física da mãe e do bebê. }\end{array}$ \\
\hline 06 & $\begin{array}{c}\text { Kair \& Colaizy } \\
\quad(2016)\end{array}$ & $\begin{array}{l}\text { Hospital } \\
\text { Pediatrics }\end{array}$ & $\begin{array}{l}\text { Estudo de } \\
\text { Coorte } \\
\text { Retrospectiv } \\
\quad 0\end{array}$ & $\begin{array}{l}\text { Suprimento inadequado do leite; } \\
\text { Preocupação materna de que o leite materno não } \\
\text { satisfaça os bebês; Dificuldades para amamentar. }\end{array}$ \\
\hline 07 & $\begin{array}{l}\text { Cruz \& } \\
\text { Sebastião } \\
(2015)\end{array}$ & $\begin{array}{l}\text { Revista Disturbios } \\
\text { da Comunicação }\end{array}$ & $\begin{array}{l}\text { Estudo } \\
\text { Transversal } \\
\text { qualitativo }\end{array}$ & $\begin{array}{c}\text { Insegurança para o sucesso da amamentação devido à } \\
\text { prematuridade do RN; Pouca experiência das mães } \\
\text { para amamentar durante o internamento na UTI Neo; } \\
\text { Angústia e sofrimento pelo fato do RN estão em uma } \\
\text { UTI; Pouco contato com o RN; Pouco tempo para } \\
\text { amamentar o RN; } \\
\text { Falta de habilidade no cuidado do RN. }\end{array}$ \\
\hline 08 & $\begin{array}{l}\text { Tronco et al. } \\
\quad \text { (2015) }\end{array}$ & $\begin{array}{c}\text { Revista de } \\
\text { Enfermagem } \\
\text { Escola Anna Nery }\end{array}$ & $\begin{array}{l}\text { Pesquisa } \\
\text { qualitativa }\end{array}$ & $\begin{array}{c}\text { Cansaço materno; Dor durante amamentação; } \\
\text { Dificuldade para ordenhar; Leite materno } \\
\text { empredrado; }\end{array}$ \\
\hline
\end{tabular}

Fonte: Autores (2021).

\section{Discussões}

Os medos maternos, gerados pela instabilidade clínica do RN, impõem à mãe o desafio de conviver diariamente com a possibilidade da perda do filho. Nessas circunstâncias, a sobrevivência do bebê assume toda a sua atenção e outras questões passam a ser secundárias, principalmente em relação à amamentação e seu próprio bem- estar e, por isso, a hospitalização de um bebê prematuro é tão difícil para elas. Muitas delas definem essa situação como um tormento e um momento de estresse e 
algo que deve ser esquecido ao longo do tempo. Além disso, todo este impacto na vida materna pode determinar, inclusive, no desmame precoce (Pereira et al., 2015).

Conforme Veronez et al. (2017) afirma, as alterações no quadro clínico do bebê contribuem para que surjam momentos de angústia e apreensão, bem como a sensação de impotência e incerteza. Desta forma, é importante ressaltar que o vínculo de confiança entre a família e a equipe de profissionais deve ser construído num processo de comunicação constante e efetiva. Já Pereira et al. (2015) trazem em seu estudo que os sentimentos ruins e a vivência dessas mães dentro de uma UTIN faz com que elas considerem a amamentação como algo muito complexo e difícil de lidar, contrariando toda sua construção social em relação ao ato de amamentar, definindo-a como um trabalho que exige treinamento e conhecimento.

Nesse contexto, foram identificados sentimentos negativos, como tristeza, insegurança, dúvida, impotência/incapacidade e até mesmo o sentimento de culpa causado pelo impedimento imediato de amamentar o filho em consequência do processo de internação (Amando et al., 2016). Independente da experiência de um período conturbado, lidar com a distância física, conviver em um ambiente complexo, estressante e, somente conseguir amamentá-los após um período de tempo considerável, algumas mães exteriorizam sensação de alegria, felicidade e satisfação quando vivenciaram o amamentar após um período de dificuldade (Amando et al., 2016).

O ato de amamentar em uma UTIN não é tão simples. De acordo com Pereira et al. (2015), algumas restrições dificultam tal prática, onde a mãe passa a necessitar da ajuda de um profissional para estabelecer a mamada, a sucção inefetiva é uma delas. Devido ao uso de dispositivo e baixo estimulo da sucção ao nascerem, os RNs têm dificuldade para realizar a pega corretamente e sugar o leite materno, tornando-se barreiras para a amamentação. Além disso, os RNs prematuros hospitalizados apresentam um padrão de sucção diferente de uma criança a termo, podendo ser mais lento e profundo, o que causa uma preocupação nas mães com relação ao ganho de peso do seu filho.

Outro fator que interfere diretamente na amamentação é a estrutura mamária da mulher. A apresentação do mamilo, aparição de fissuras mamilares, dores e mastites devem ser identificadas desde o pré-natal para que não se tornem empecilho durante a amamentação, visto que as queixas de dor ao aleitar são comuns entre as puérperas (Teston et al., 2018).

$\mathrm{O}$ ambiente hospitalar muitas das vezes se torna uma barreira para àquela mãe que deseja amamentar o seu RN. Por ser um ambiente complexo e rodeado de tecnologias, a UTIN dificulta o contato espontâneo do binômio mãe-bebê complicando ainda mais a prática da amamentação. Amando et al. (2016) retratam que além de ser considerado um local estressante devido a rotina do próprio setor, o uso contínuo de aparelhos pelo RN, além de impedir à amamentação, causa bastante ansiedade na mãe, o que diminui a saída do leite.

Para muitas mulheres é necessária certa experiência para amamentar. O pouco tempo que é permitido às mães para estarem com os seus filhos devido à rotina do setor ocasiona a falta dessa prática no aleitamento, juntamente com a insegurança, justificada pela ausência de habilidades por classificá-los como um ser muito pequeno e frágil (Cruz \& Sebastião, 2015). A enfermagem possui um papel fundamental quando diz respeito ao incentivo ao aleitamento materno e isso se inicia na atenção básica onde o apoio, orientação e encorajamento auxiliam no desejo de iniciar e manter a amamentação (Barbieri $e t$ al., 2015).

Segundo Azevedo et al. (2015), é imprescindível que o enfermeiro possua o conhecimento técnico e científico sobre a anatomia e fisiologia da lactação, para assim orientar a mãe sobre o posicionamento adequado, pega correta, extração manual do leite e dentre outras alternativas que favoreçam este processo tão benéfico para a criança. Sendo assim, Silva et al (2018) dizem ser de grande valia que esse acolhimento seja realizado, pois cria na mulher um sentimento de confiança e auto capacidade frente aos desafios, deixando-as motivadas e esperançosas para oinício da amamentação.

Além disso, visto a era digital em que vivemos e como as gestantes utilizam a internet como meio de informação, fazse necessário que os profissionais disponibilizem fontes seguras de informações como complemento nas orientações, para 
assim minimizar os possíveis danos causados por informações errôneas vistas na internet (Azevedo et al., 2015). Entretanto, é observado um obstáculo para eficácia desse incentivo e, isto ocorre quando os profissionais que lidam com as mães na atenção primária possuem um déficit no conhecimento acerca dos benefícios da amamentação ou, então, os possuem, mas não realizam estratégias e aconselhamentos para conscientizar as gestantes sobre a importância de manter o aleitamento materno, mesmo que em situações de adversidades (Machado \& Lara, 2018).

Quando ocorre um nascimento pré-termo e o RN necessita ser encaminhado pra uma Unidade de Terapia Intensiva, o distanciamento físico, os aparelhos, os cateteres e soros formam uma barreira para o contato entre a mãe e o bebê, e mesmo que durante toda a gestação houvesse o desejo de amamentar, a nutriz pode se encontrar frustrada e se sentindo incapaz diante desse novo cenário, sendo então necessário, mais uma vez, que o enfermeiro se torne um facilitador e mediador, auxiliando e estimulando a reconstrução do vínculo que foi quebrado, para que mesmo cansada e triste com toda situação em que se encontra, tenha forças para continuar firme ao lado do seu filho (Coutinho \& Kaiser, 2015).

É importante ressaltar que o apoio não deve se restringir apenas ao binômio mãe e filho, o(a) companheiro(a) necessita também de um olhar especial, permitindo que ele(a) acompanhe e participe das rotinas do seu filho durante o internamento, fortalecendo assim o vínculo e capacitando esse(a) companheiro(a) para auxiliar a nutriz (de Andrade Almeida $\&$ da Silva Diniz, 2016).

A angústia vivenciada pelas mães de prematuros se estende muito além dos corredores da UTI. Muitas estão distantes de seus entes queridos desde o parto e até com outros filhos em casa para se preocupar, e em muitos casos sem condição financeira para ir e vir ao hospital. É a partir daí que a família deve se permitir ser uma aliada mesmo que a distância, se comprometendo a apoiar e ajudar no que for possível e para que a estadia no hospital seja a melhor possível, sendo então a primeira e mais importante rede de apoio que ela poderá ter para seguir firme durante a recuperação desse bebê (Tronco et al., 2015).

\section{Considerações Finais}

Tendo em vista os aspectos observados, e a importância que o leite materno tem na vida de uma criança, foi possível observar que em um cenário de UTIN existe uma fragilidade com relação ao aleitamento materno evidenciado por barreiras que dificultam essa troca de nutrientes e afeto, sendo elas: a falta de apoio dos profissionais, ansiedade, exaustão, frustação, desconforto e preocupação materna, além do ambiente hostil e separação do binômio por conta do quadro clínico apresentado pelo RN. O profissional que lida com essa família precisa ter um olhar cuidadoso, atendendo as necessidades não só assistenciais, mas emocionais, sendo um facilitador, dando apoio e incentivando para que essa mulher mesmo com todas as dificuldades no percurso da internação, siga firme ao lado do seu filho. Diante disso se faz necessários novos trabalhos a fim de melhorar as políticas de amamentação para recém-nascidos internados na UTIN.

\section{Referências}

Alves, E., Magano, R., Amorim, M., Nogueira, C., \& Silva, S. (2016). Factors influencing parent reports of facilitators and barriers to human milk supply in neonatal intensive care units. Journal of Human Lactation, 32(4), 695-703.

Amando, A. R., Tavares, A. K., de Oliveira, A. K. P., Fernandes, F. E. C. V., Sena, C. R. S., \& Melo, R. A. (2016). Percepção de mães sobre o processo de amamentação de recém-nascidos prematuros na Unidade Neonatal. Revista Baiana de Enfermagem30,(4).

Azevedo, A. R. R., Alves, V. H., Souza, R. D. M. P. D., Rodrigues, D. P., Branco, M. B. L. R., \& Cruz, A. F. D. N. D. (2015). O manejo clínico da amamentação: saberes dos enfermeiros. Escola Anna Nery, 19(3), 439-445.

Barbieri, M. C., Bercini, L. O., de Melo Brondani, K. J., Ferrari, R. A. P., Tacla, M. T. G. M., \& Sant'anna, F. L. (2015). Aleitamento materno: orientações recebidas no pré-natal, parto e puerpério. Semina: Ciências Biológicas e da Saúde, 36(1Supl), 17-24.

Brasil. (2015). Saúde da criança: aleitamento materno e alimentação complementar. Ministério da Saúde. 
Research, Society and Development, v. 10, n. 8, e39010817190, 2021

(CC BY 4.0) | ISSN 2525-3409 | DOI: http://dx.doi.org/10.33448/rsd-v10i8.17190

Brasil. (2019). Guia alimentar para crianças brasileiras menores de 2 anos. Ministério da Saúde.

Bujold, M., Feeley, N., Axelin, A., Cinquino, C., Dowling, D., \& Thibeau, S. (2018). Expressing human milk in the NICU. Advances in Neonatal Care, 18(1), $38-48$.

Coutinho, S. E., \& Kaiser, D. E. (2015). Visão da enfermagem sobre o aleitamento materno em uma unidade de internação neonatal: relato de experiência. Boletim Científico de Pediatria-Vol, 4(1), 11.

Cruz, M. R., \& Sebastião, L. T. (2015). Amamentação em prematuros: conhecimentos, sentimentos e vivências das mães. Distúrbios da Comunicação, 27(1).

de Andrade Almeida, S., \& da Silva Diniz, S. O. (2016). Os Sentimentos E As Dificuldades Do Pai De Um Filho Prematuro Internado Na Uti Neonatal. Revista Rede de Cuidados em Saúde, 10(2).

de Souza Baptista, S., Alves, V. H., de Souza, R. D. M. P., Rodrigues, D. P., da Cruz, A. F. D. N., \& Branco, M. B. L. R. (2015). Manejo clínico da amamentação: atuação do enfermeiro na unidade de terapia intensiva neonatal. Revista de Enfermagem da UFSM, 5(1), 23-31.

Gianni, M. L., Bezze, E. N., Sannino, P., Baro, M., Roggero, P., Muscolo, S., ... \& Mosca, F. (2018). Maternal views on facilitators of and barriers to breastfeeding preterm infants. BMC pediatrics, 18(1), 1-7.

Kair, L. R., \& Colaizy, T. T. (2016). Breastfeeding continuation among late preterm infants: barriers, facilitators, and any association with NICU admission? Hospital pediatrics, 6(5), 261-268.

Lima, A. P. E., Castral, T. C., Leal, L. P., Javorski, M., Sette, G. C. S., Scochi, C. G. S., \& de Vasconcelos, M. G. L. (2019). Aleitamento materno exclusivo de prematuros e motivos para sua interrupção no primeiro mês pós-alta hospitalar. Revista Gaúcha de Enfermagem, 40.

Lopes, A. M., da Silva, G. R. F., da Rocha, S. S., Avelino, F. V. S. D., \& Soares, L. S. (2015). Amamentação em prematuros: Caracterização do binômio mãefilho e autoeficácia materna. Revista Brasileira em Promoção da Saúde, 28(1), 32-43.

Machado, P. Y., \& Lara, A. N. O. (2018). Estratégias De Incentivo Ao Aleitamento Materno Realizadas Pelos Enfermeiros Da Atenção Primária. InteraçãoRevista de Ensino, Pesquisa e Extensão, 20(1), 232-251.

Marson, A. P. (2015). Implantação da $1^{a}$ etapa do Método Canguru em uma Unidade Neonatal: uma análise dos benefícios e dificuldades.

Morais, A. C., Guirardi, S. N., \& Miranda, J. D. O. F. (2020). Práticas de aleitamento materno em unidade de terapia intensiva neonatal. Revista Baiana de Enfermagem 34, .

Pereira, L. B., Abrão, A. C. F. D. V., Ohara, C. V. D. S., \& Ribeiro, C. A. (2015). Vivências maternas frente às peculiaridades da prematuridade que dificultam a amamentação. Texto \& Contexto-Enfermagem, 24(1), 55-63.

Rossman, B., Meier, P. P., Janes, J. E., Lawrence, C., \& Patel, A. L. (2017). Human milk provision experiences, goals, and outcomes for teen mothers with low-birth-weight infants in the neonatal intensive care unit. Breastfeeding Medicine, 12(6), 351-358.

Silva, D. D. D., Schmitt, I. M., Costa, R., Zampieri, M. D. F. M., Bohn, I. E., \& Lima, M. M. D. (2018). Promoção do aleitamento materno no pré-natal: discurso das gestantes e dos profissionais de saúde. Revista Mineira de Enfermagem, 22, 1-9.

Souza, M. T. D., Silva, M. D. D., \& Carvalho, R. D. (2010). Revisão integrativa: o que é e como fazer. Einstein, 8(1), 102-106.

Teston, E. F., Reis, T. S., de Góis, L. M., Spigolon, D. N., Maran, E., \& Marcon, S. S. (2018). Aleitamento materno: percepção do pai sobre seu papel. Revista de Enfermagem do Centro-Oeste Mineiro, 8.

Tronco, C. S., Padoin, S. M. D. M., Paula, C. C. D., Rodrigues, A. P., Neves, E. T., \& Weinmann, A. R. M. (2015). Manutenção da lactação de recém-nascido pré-termo: rotina assistencial, relação mãe-filho e apoio. Escola Anna Nery, 19(4), 635-640.

Valadares, C. (2016). Brasil é referência mundial em amamentação. Brasília-DF: Portal da Saúde.

Veronez, M., Borghesan, N. A. B., Corrêa, D. A. M., \& Higarashi, I. H. (2017). Vivência de mães de bebês prematuros do nascimento a alta: notas de diários de campo. Revista Gaúcha de Enfermagem, 38(2).

Yang, Y., Brandon, D., Lu, H., \& Cong, X. (2019). Breastfeeding experiences and perspectives on support among Chinese mothers separated from their hospitalized preterm infants: a qualitative study. International breastfeeding journal, 14(1), 1-7. 